\title{
The 'GO' system - a novel method of microculture for in vitro development of mouse zygotes to the blastocyst stage
}

\author{
G. A. Thouas, G. M. Jones and A. O. Trounson \\ Monash Institute of Reproduction and Development, Monash University, Monash Medical Centre, \\ 246 Clayton Road, Clayton, Victoria 3168, Australia
}

\begin{abstract}
A novel system of in vitro culture termed the 'glass oviduct' or ' $\mathrm{GO}^{\prime}$ ' culture system is described. Mouse zygotes were cultured in pairs to the blastocyst stage in open-ended $1 \mu \mathrm{l}$ glass capillaries. ' $\mathrm{GO}$ ' culture supported the development of significantly more hatching or hatched blastocysts than did a standard microdroplet (10 zygotes per $20 \mu \mathrm{l}$ ) control culture (48.3 versus $3.3 \%$, respectively). ' $\mathrm{GO}$ ' blastocysts contained significantly larger populations of cells (92 \pm 3 versus $75 \pm 3)$, and inner cell mass $(25 \pm 1$ versus $21 \pm 1)$ and trophectoderm ( $68 \pm 2$ versus $53 \pm 3$ ) subpopulations, compared with microdroplet-derived blastocysts. Before blastulation, ' $G O^{\prime}$-derived morulae were found to contain significantly more cells than microdroplet-derived morulae $(27 \pm 0.7$ versus $14 \pm 0.5)$. After implantation, ' $G O^{\prime}$ blastocysts formed fetuses at a similar rate to microdropletderived blastocysts (55 versus $62 \%$ ), but at a lower rate than blastocysts derived in vivo $(80 \%)$. ' $\mathrm{GO}^{\prime}$ - and microdroplet-derived fetuses were similar in wet weight to each other (0.412 and $0.415 \mathrm{~g}$, respectively) but were heavier than fetuses derived from flushed blastocysts $(0.390 \mathrm{~g})$. An additional experiment investigated whether the beneficial
\end{abstract}

effect of ' $G \mathrm{O}$ ' culture was due to the significantly increased embryo density. Proportions of hatching or hatched blastocysts after ' $\mathrm{GO}^{\prime}$ ' culture $(\mathbf{5 0} \%)$ were higher than after standard microdroplet culture $(7.6 \%)$, but were not different from culture in high embryo density microdroplets (20 zygotes per $10 \mu \mathrm{l} ; 42 \%$ ). 'GO' blastocysts contained more cells $(79.6 \pm 2.1)$ than did standard microdropletderived blastocysts $(68.7 \pm 2.0)$, but were similar to high density microdroplet-derived blastocysts (85.8 \pm 2.7$)$. Similarly, ' $G O^{\prime}$ blastocysts contained more trophectoderm cells $(62.2 \pm 2.0)$ than did standard microdroplet-derived blastocysts $(52.7 \pm 1.7)$, but were similar to the high density microdroplet blastocysts $(68.8 \pm 2.5)$. Numbers of inner cell mass cells ('GO', standard microdroplet and high density microdroplet culture) were not different from each other $(17.4 \pm 0.5,16 \pm 0.5$ and $17 \pm 0.4$, respectively). In conclusion, the ' $G \mathrm{O}^{\prime}$ ' culture system represents an alternative method to the microdroplet system for small numbers of preimplantation embryos, without detriment to implantation potential.

\section{Introduction}

Recent research investigating the in vitro culture of mammalian cleavage stage embryos to the blastocyst stage of development has been primarily focused on the improvement of culture medium composition to satisfy stage-specific nutrient requirements and to overcome culture-induced blocks to development (Bavister, 1995; Gardner and Lane, 1997; Gardner, 1998a,b). Few studies have addressed the role of the physical environment that contains or encloses the culture medium, which together with the incubation conditions represents an integral component of the overall culture system.

Mammalian preimplantation embryos have traditionally been cultured from the one-cell or two-cell stage to the blastocyst stage in various volumes of culture medium

Email: George.Thouas@med.monash.edu.au within a variety of sterile glass or disposable plastic culture vessels (for a review, see Brinster, 1968). Several modifications to these static environments have been investigated for their effects on development of preimplantation embryos in vitro. The inclusion of a monolayer of confluent epithelium in co-culture with preimplantation embryos has been one such modification. The beneficial effects of co-culture on embryo development have been attributed to the release of embryotrophic factors into the culture medium (positive conditioning) or the removal of deleterious components from the culture medium (negative conditioning) (Bongso and Fong, 1993; Bongso et al., 1993, 1994). Despite the claims of the putative benefits of co-culture on embryo development in vitro, several prospective, randomized studies have failed to demonstrate a significant difference in human blastocyst development and pregnancy rates when co-culture and cell-free culture have been compared (Van Blerkom, 1993; Sakkas et al., 1994; Magli et al., 1995), particularly 
when the newer stage-specific culture media are used (Fong et al., 1998).

Dynamic perfusion culture systems that use a continuous inflow and outflow of fresh medium have also been used for development of mammalian preimplantation embryos in vitro with equivocal results (Pincus and Werthessen, 1938; Pruitt et al., 1991; Wilson et al., 1992). The main disadvantage of such systems is the progressive depletion of putative embryotrophic autocrine/ paracrine factors secreted into the culture environment by the developing embryo (Paria and Dey, 1990; Lane and Gardner, 1992; Gardner et al., 1994) that would normally be allowed to accumulate in static culture systems.

Recently, a modification to traditional static culture, the Well of the Well (WOW) culture system, has been demonstrated to improve development of bovine embryos to the blastocyst stage in vitro (Vajta et al., 2000). The WOW culture system is created by burning Vshaped or cylindrical holes approximately $300 \mu \mathrm{m}$ in depth in the base of a well of a four-well culture dish. The main difference between the WOW culture system and traditional culture in wells, tubes or microdroplets of medium under oil is the significant reduction in the volume of culture medium surrounding the embryo. Vajta et al. (2000) suggested that the physiological benefit of the WOW culture system might be that putative autocrine factors remain concentrated in the immediate microenvironment of the embryo, which also remains sufficiently bathed in a relatively large pool of nutrient medium from above.

Mulnard (1965) described a similar modification to static culture systems that also uses reduced volumes of culture medium. Small glass capillary tubes with an internal diameter of 1-2 $\mathrm{mm}$ were used to contain mouse embryos that were cultured horizontally on the bottom of a Petri dish with an oil overlay. There have been no more recent reports of this method as a viable alternative to culture of mammalian embryos in tubes, dishes or microdroplets of medium under oil. However, glass capillaries or small plastic straws have been used for fertilization of human oocytes in vitro (van der Ven et al., 1989; Ranoux and Seibel, 1990; Hammitt et al., 1991) and to culture mouse two-cell embryos in precise volumes of medium in a study of embryo density in vitro (Lane and Gardner, 1992).

In the present study, a novel system of microculture termed the ' $\mathrm{GO}$ ', or 'glass oviduct', culture system is described, in which mouse zygotes are cultured vertically within open-ended, sterile, glass microcapillaries to the blastocyst stage of development in $1 \mu$ l of a simple, bicarbonate-buffered medium supplemented with albumin. The effect of this microenvironment on embryo cleavage, blastocyst expansion and blastocyst hatching in vitro, and on implantation and fetal normality after blastocyst transfer to pseudopregnant recipients was investigated and compared with outcomes after more traditional culture in microdroplets of culture medium under oil.

\section{Materials and Methods}

Animals

$\mathrm{C} 57 \mathrm{BL} 6 \times \mathrm{CBA} / \mathrm{Ca} \mathrm{F} 1$ (hybrid) female and male mice were used for all experiments. Mice were housed in groups or pairs in an environmentally controlled room at $22-24^{\circ} \mathrm{C}$ with a $12 \mathrm{~h}$ light: $12 \mathrm{~h}$ dark photoperiod. Pelleted food and water were available ad libitum.

For zygote collection and culture, 4-6-week-old female mice were induced to superovulate by i.p. injection of 5 iu equine chorionic gonadotrophin (eCG) (Folligon; Intervet Laboratories Ltd, Bendigo), followed approximately $48 \mathrm{~h}$ later by an i.p. injection of 5 iu hCG (Chorulon; Intervet Laboratories Ltd). Female mice were placed with 12-week-old male mice (one female per male) overnight to mate naturally. Mating was checked the morning after by the presence of a vaginal plug. At approximately $22 \mathrm{~h}$ (after hCG administration), the 'plugged' female mice were killed by cervical dislocation and dissected to obtain their oviducts. The isolated oviducts were incubated in pre-warmed Hepes-buffered mouse tubal fluid medium (Hepes-MTF) (Gardner and Leese, 1990) supplemented with $3 \mathrm{mg} \mathrm{BSA} \mathrm{ml}^{-1}$ (Gibco BRL, Grand Island, NY).

For intrauterine blastocyst transfer experiments, 7-12week-old female mice were used as pseudopregnant recipients. Female mice were mated with 12-weekold vasectomized males. Females were considered as pseudopregnant by the presence of a vaginal plug as described for superovulated females and were then housed for a further 2 days after mating before being used as embryo recipients. Pseudopregnant females underwent general anaesthesia by i.p. injection of up to $0.5 \mathrm{ml}$ Avertin (prepared as described by Hogan et al., 1986) using 222-tribromoethanol and tertiary amyl alcohol (Sigma-Aldrich P/L, Sydney). Anaesthesia was confirmed by footpad reflex. During surgery, anaesthetized females were monitored every 5 min for signs of arousal, in which case an additional $0.05 \mathrm{ml}$ anaesthetic was administered. After surgery, the females were housed in boxes incubated on a $37^{\circ} \mathrm{C}$ warming pad. The females were monitored every 10 min until they aroused and thereafter they were monitored once a day for a further 15 days. The mice were killed by cervical dislocation for analysis of fetal development. Conceptuses were dissected from uterine horns and washed in warmed Hepes-MTF supplemented with $3 \mathrm{mg} \mathrm{BSA} \mathrm{ml}^{-1}$. Microscopic analysis of normality and mass measurement were performed, and the conceptuses were killed by decapitation.

\section{Ethics of experimentation}

The present study was approved by Monash Medical Centre Animal Ethics Committee A, Monash Medical 
Centre, Clayton, Victoria, Australia, approval numbers A1198/25 and A2000/11. All experiments were conducted in accordance with the $1997 \mathrm{NH} \&$ MRC Australian Code of Practice for the care and use of animals for scientific purposes and the Victorian Prevention of Cruelty to Animals Act and Regulations, 1986.

\section{Zygote collection and culture}

In vivo fertilized cumulus-enclosed zygotes were isolated from the oviducts according to the method described by Gardner and Lane (1993). Cumulus-enclosed zygotes were incubated briefly in Hepes-MTF containing 50 iu hyaluronidase $\mathrm{ml}^{-1}$ (Sigma Chemical Co., St Louis, $\mathrm{MO}$ ). The cumulus was removed by gentle pipetting and the zygotes were washed three times in Hepes-MTF

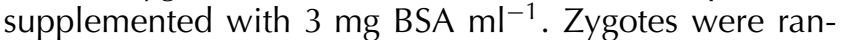
domly distributed between test and control $(20 \mu \mathrm{l})$ microdroplets of pre-equilibrated bicarbonate-buffered MTF supplemented with $4 \mathrm{mg} \mathrm{BSA} \mathrm{ml}^{-1}$ under light mineral oil (Sigma). Ten zygotes were allocated to each $20 \mu \mathrm{l}$ microdroplet, as this version of microdroplet culture represents the standard, established in-house culture method. A minimum of 30 zygotes for each test and control replicate, and a minimum of three replicates, were performed for each experiment. Test zygotes were aspirated by capillary action into glass capillaries (200 $\mu \mathrm{m}$ internal diameter, $1 \mu \mathrm{l}$ capacity; Drummond Scientific Company, Broomall, PA) at a density of two zygotes per capillary. The capillaries were then inverted and incubated vertically throughout the culture period. The capillaries had been soaked in $3 \%(\mathrm{v} / \mathrm{v}) \mathrm{HCl}$ for $24 \mathrm{~h}$, rinsed and soaked in Milli-Q water for $24 \mathrm{~h}$, and sterilized by dry heat $\left(120^{\circ} \mathrm{C}\right.$ for $\left.24 \mathrm{~h}\right)$ before use. Control zygotes remained in the microdroplets for the entire period of culture. In addition to the positive control, a negative control to test the culture medium, culture disposables and incubation conditions was included for each experiment. For this control, groups of 20 zygotes (per microdroplet) were cultured using identical disposables and culture conditions in $20 \mu \mathrm{l}$ microdrops of MTF without protein supplementation. A minimum of $70 \%$ zygote development to the blastocyst stage by day 4.5 in vitro was accepted as a pass for in vitro conditions. All zygotes were pooled from consecutive groups of five mice before random distribution into each replicate group before culture. All zygotes and embryos were cultured in a $\mathrm{CO}_{2}$ controlled, temperature-regulated incubator (Heraeus Instruments $\mathrm{GmbH}$, Hanau) at $37^{\circ} \mathrm{C}$ in a humidified atmosphere of $5 \% \mathrm{CO}_{2}$ in air.

The above zygote allocation was repeated with the inclusion of a second experimental control group (high density group) comprising 20 zygotes in $10 \mu \mathrm{l}$ microdroplets to control for the potential effects of zygote density on embryo development. The embryo density of this group was two zygotes per $\mu l$, which matches the density of zygotes in the 'GO' culture system.
Fluorescent nuclear staining and assessments of number of cells

Blastocysts were differentially stained for numbers of inner cell mass (ICM) and trophectoderm cells using DNA-specific fluorochromes according to a previous method described by Thouas et al. (2002). After staining, whole blastocysts were mounted in glycerol (Sigma-Aldrich P/L) on glass microscope slides and flattened under a coverslip. For determination of numbers of blastocyst ICM and trophectoderm cells, the slides were visualized using an inverted microscope (Leica DMR; Leica Microsystems Wetzlar GmbH, Germany) fitted with ultraviolet illumination and excitation filters (460 $\mathrm{nm}$ for blue and red fluorescence, and $560 \mathrm{~nm}$ for red only).

For determination of total number of cells, morulae were fixed and stained in a solution of $25 \mu \mathrm{g}$ bisbenzimide $\mathrm{ml}^{-1}$ (Sigma) in 100\% ethanol and mounted in glycerol as described previously. Whole mounts were visualized under a microscope as described for determination of numbers of ICM and trophectoderm cells, except that only the blue (460 nm) excitation filter was used.

\section{Intrauterine blastocyst transfer and fetal assessment}

Mouse blastocysts that had formed approximately $96 \mathrm{~h}$ after culture in microdroplets or capillaries were transferred into the uterine horns of day 2.5 pseudopregnant female mice (Whitten and Biggers, 1968). As an additional control, in vivo derived blastocysts were isolated from the uterine horns of superovulated females at day 3.5 after mating, washed in Hepes-MTF supplemented with $3 \mathrm{mg} \mathrm{BSA} \mathrm{ml}^{-1}$ and transferred on the same day. At the conclusion of culture, blastocysts from 'GO' culture were pooled by evacuation into fresh preequilibrated microdroplets of MTF supplemented with $4 \mathrm{mg} \mathrm{BSA} \mathrm{ml}^{-1}$. Blastocysts that were partially expanded, fully expanded, partially hatched or fully hatched were chosen for transfer at random from microdroplet or 'GO' cultures. A maximum of six blastocysts was allocated per uterine horn. A total of approximately 200 blastocysts from each control group (in vivo derived or in vitrocultured in microdroplets) and from the test group (in vitro cultured in ' $\mathrm{GO}$ ') were transferred. On day 15.5 after mating, pregnant foster mice were killed and their uterine horns were assessed for the presence of a conceptus or an implantation scar. The fetuses were isolated, washed in warmed Hepes-MTF supplemented with $3 \mathrm{mg}$ BSA $\mathrm{ml}^{-1}$, assessed visually for physical normality and weighed.

\section{Endpoint measurements}

Endpoints of the culture included: the proportion of zygotes that formed blastocysts; the proportion of zygotes 


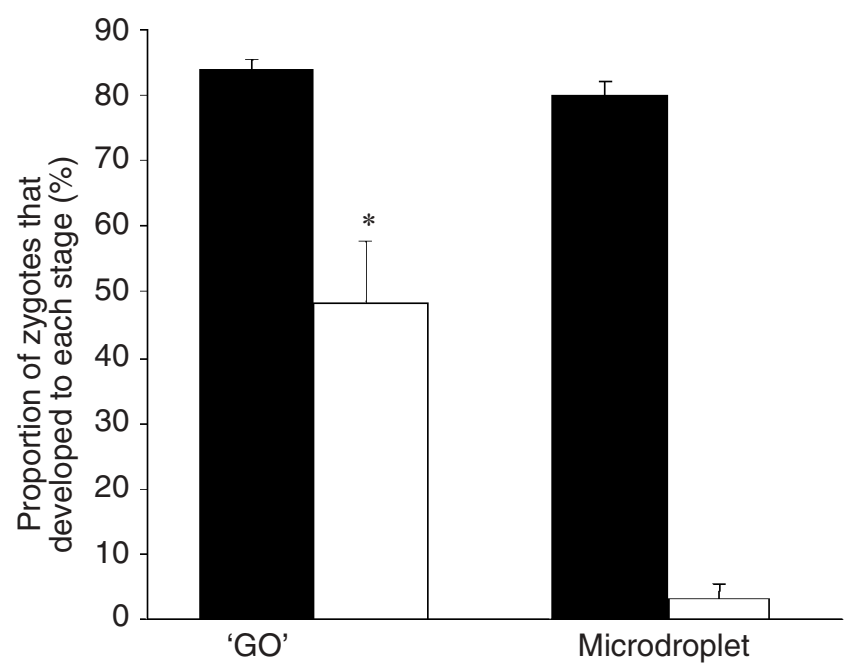

Fig. 1. Development of mouse blastocysts after culture from the zygote stage in two different systems: the 'GO' (glass oviduct) system and the microdroplet system (control). Total proportions of zygotes cultured from three replicates ( $n=90$ zygotes) that developed to the blastocyst (ם) and hatching/hatched blastocyst $(\square)$ stages. *Significant difference compared with corresponding microdroplet culture value $(P<0.0001)$.

that formed partially hatched or fully hatched blastocysts (three replicates of 30 zygotes per replicate); the number of cells in the ICM and trophectoderm, and the total cell populations of the blastocysts generated. An additional endpoint was conducted to determine the kinetics of embryo development. Approximately 80 zygotes (four replicates of 20 zygotes) were isolated from $20 \mu \mathrm{l}$ microdroplet (control) and ' $\mathrm{GO}$ ' (test) cultures at approximately $72 \mathrm{~h}$ after culture, and stained for total cell counts as an indication of cell proliferation before phenotypic differentiation. This endpoint was not included for the additional embryo density experiment due to time constraints.

Implantation rate was determined as the mean number of blastocysts that implanted and were either resorbed before day 14.5 after mating or formed a normal fetus, and was expressed as a percentage of the total number of blastocysts transferred. It was assumed that blastocysts not accounted for by either an implantation scar or a conceptus had not implanted. The fetuses were all weighed as wet specimens.

\section{Statistical analysis}

The results from the three replicate experiments are presented as the mean \pm SEM of the proportions of zygotes that developed to the blastocyst or hatched blastocyst stage in the 'GO' and microdroplet cultures and were analysed by Fisher's exact test. The numbers of cells in the ICM and trophectoderm, and the total cell populations of blastocysts grown in the 'GO' or microdroplet cultures are expressed as the mean \pm SEM for the three replicate experiments, and were transformed

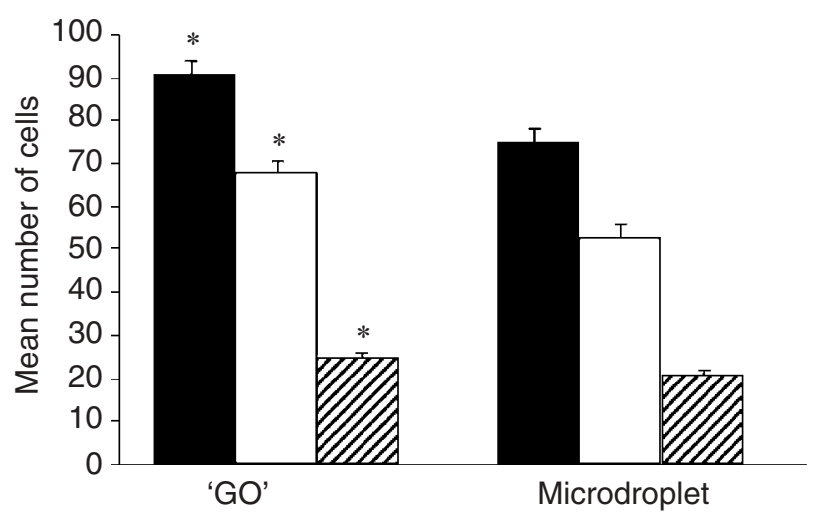

Fig. 2. Mean cell populations of mouse blastocysts cultured from zygotes in the 'GO' (glass oviduct) system and the microdroplet system (control). Total number of cells ( $\mathbf{\square})$, and numbers of trophectoderm ( $\square$ ) and inner cell mass (龌) cells, are shown. *Significant difference compared with corresponding microdroplet culture value $(P<0.01)$.

and compared using Tukey's ANOVA. For the kinetic endpoint, the total number of cells of morulae grown in 'GO' and microdroplet control cultures are expressed as the mean \pm SEM of the three replicates and were analysed by the Mann-Whitney test for non-parametric data.

Mean numbers of fetuses, resorptions or failed implantations (losses) are expressed as proportions of the total number of transferred blastocysts that were in vivo derived or cultured in ' $\mathrm{GO}$ ' or microdroplet cultures. These proportions were compared individually using the chi-squared test. The mean masses of fetuses formed from in vivo derived and in vitro derived ('GO' and microdroplet) blastocysts were compared by ANOVA. $P<0.05$ was considered significant for all endpoint measurements.

\section{Results}

\section{Blastocyst formation, hatching and cell populations}

The proportion of zygotes that developed to the blastocyst stage in 'GO' cultures was not different from that in standard microdroplet control cultures 84 and $80 \%$, respectively; Fig. 1). However, the proportion of zygotes that developed to the partially or fully hatched blastocyst stage in the 'GO' system was significantly higher than for standard culture in microdroplets (48.3 and 3.3\%, respectively; $P<0.0001)$. The mean number of cells in blastocysts cultured in the 'GO' system was higher than that of blastocysts cultured in standard microdroplet control cultures $(92 \pm 3$ versus $75 \pm 3 ; P<0.0001)$. Furthermore, ' $\mathrm{GO}$ ' blastocysts had larger subpopulations of ICM cells $(25 \pm 1 ; P<0.01)$ and trophectoderm cells $(68 \pm 2 ; P<0.001)$ than did control blastocysts $(21 \pm 1$ and $53 \pm 3$, respectively; Fig. 2). 
Table 1. Effect of culture treatment on the viability of mouse blastocysts after intrauterine transfer

\begin{tabular}{lcccc}
\hline Treatment & Fetuses $(\%)$ & Resorptions $(\%)$ & Losses $(\%)$ & Mean fetal mass $(\mathrm{mg})$ \\
\hline $\mathrm{GO}^{\prime}(n=227)$ & $55^{\mathrm{a}}(124 / 227)$ & $19^{\mathrm{a}}(43 / 227)$ & $26^{\mathrm{a}}(60 / 227)$ & $412 \pm 7^{\mathrm{a}}$ \\
Microdroplet $(n=212)$ & $62^{\mathrm{a}}(131 / 212)$ & $26^{\mathrm{a}}(54 / 212)$ & $13^{\mathrm{b}}(27 / 212)$ & $415 \pm 7^{\mathrm{a}}$ \\
In vivo control $(n=156)$ & $80^{\mathrm{b}}(124 / 156)$ & $10^{\mathrm{b}}(16 / 156)$ & $10^{\mathrm{b}}(16 / 156)$ & $390 \pm 4^{\mathrm{b}}$ \\
\hline
\end{tabular}

'GO': glass oviduct.

$n=$ number of blastocysts transferred.

${ }^{\text {ab } W i t h i n ~ a ~ c o l u m n, ~ v a l u e s ~ w i t h ~ d i f f e r e n t ~ s u p e r s c r i p t s ~ a r e ~ s i g n i f i c a n t l y ~ d i f f e r e n t ~}(P<0.05)$.

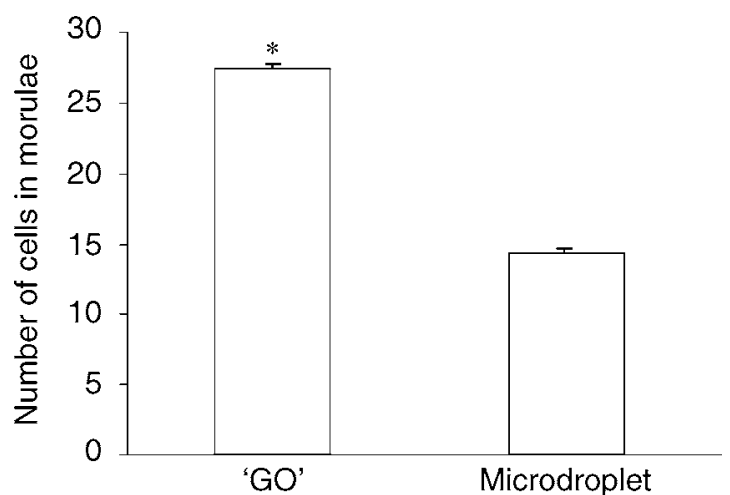

Fig. 3. Mean number of cells in morulae cultured from mouse zygotes after 3 days of culture using the 'GO' (glass oviduct) system and microdroplet system (control). *Significant difference compared with corresponding microdroplet culture value $(P<$ 0.0001).

\section{Kinetics of development of cultured embryos}

Zygotes were cultured in ' $\mathrm{GO}$ ' and standard microdroplet cultures for $72 \mathrm{~h}$ and isolated at the morula stage, before complete phenotypic differentiation occurs, to determine whether the increased mean cell populations of blastocysts grown in the 'GO' system resulted from an increased rate of cell division at earlier stages. Morulae recovered from the 'GO' system after $72 \mathrm{~h}$ of culture had a larger number of cells than did those isolated at the same time from standard microdroplet control cultures $(27 \pm 0.7$ versus $14 \pm 0.5 ; P<0.0001 ;$ Fig. 3$)$.

\section{Post-implantation development}

The results of post-implantation development are shown (Table 1). Fetal formation of transferred blastocysts grown in the 'GO' system (55\%) was not different from that of blastocysts grown in standard microdroplets (62\%); however, in vivo-derived transferred blastocysts formed significantly more fetuses $(80 \%)$ than did either 'GO'- $(P<0.005)$ or standard microdroplet-derived $(P<$ $0.0001)$ blastocysts. Fetuses derived from blastocysts grown in the 'GO' system isolated on day 14.5 after mating were of similar mean mass $(412 \mathrm{mg})$ to those grown in standard microdroplets $(415 \mathrm{mg})$; however,

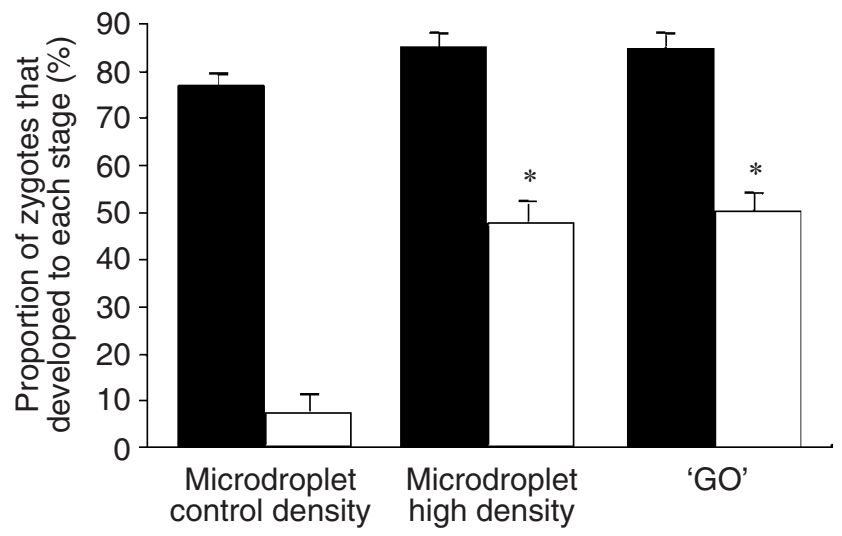

Fig. 4. Development of mouse blastocysts after culture from the zygote stage in two different systems: the 'GO' (glass oviduct) system and the microdroplet system (control density: 10 zygotes per $20 \mu \mathrm{l}$ droplet; high density: 20 zygotes per $10 \mu \mathrm{l}$ droplet). Total proportions of zygotes cultured from three replicates that developed to the blastocyst (ם) and hatching/hatched blastocyst $(\square)$ stages. *Significant difference compared with corresponding control microdroplet culture value $(P<0.0001)$.

in vivo derived blastocysts formed significantly lighter fetuses $(390 \mathrm{mg})$ than did blastocysts from either in vitro group $(P<0.01)$.

Fetal resorption of transferred blastocysts grown in the 'GO' system (19\%) was no different from that of blastocysts grown in standard microdroplets (26\%); however, significantly fewer in vivo derived blastocysts were resorbed $(10 \%)$ compared with blastocysts cultured in either 'GO' $(P<0.005)$ or standard microdroplets $(P<$ $0.05)$. The proportion of transferred blastocysts grown in the 'GO' system that failed to implant $(26 \%)$ was significantly higher than that of blastocysts grown in standard microdroplets $(13 \% ; P \leqslant 0.0005)$ or in vivoderived blastocysts $(10 \% ; P<0.0005)$.

\section{Blastocyst formation, hatching and cell populations at variable zygote densities}

The proportion of zygotes that developed to the blastocyst stage in 'GO' cultures was not different from that of $20 \mu \mathrm{l}$ (standard) or $10 \mu \mathrm{l}$ microdroplet (high density) control cultures (85, 77 and 85\%, respectively; Fig. 4). 


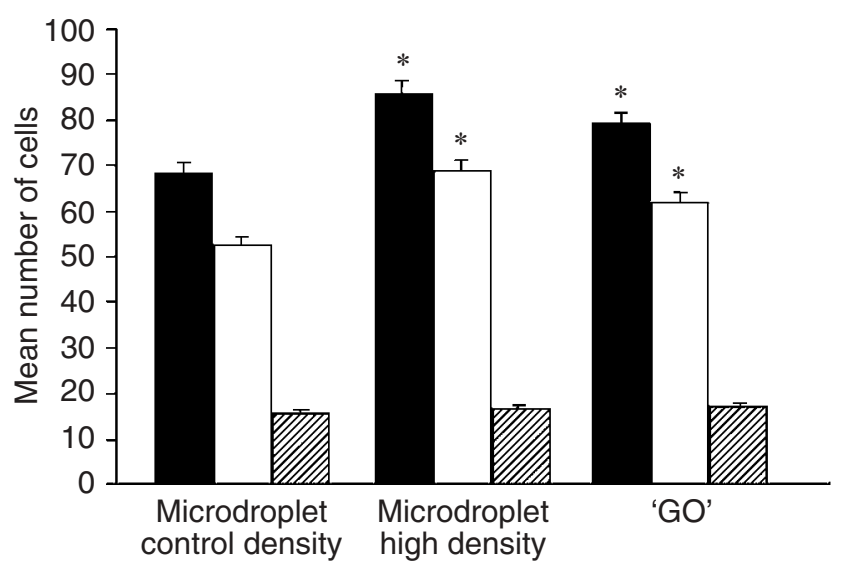

Fig. 5. Mean cell populations of mouse blastocysts cultured from zygotes in the 'GO' (glass oviduct) system and the microdroplet system (control density: 10 zygotes per $20 \mu \mathrm{l}$; high density: 20 zygotes per $10 \mu \mathrm{l})$. Total number of cells $(\mathbf{\square})$, and numbers of trophectoderm ( $\square$ ) and inner cell mass (濯) cells, are shown. *Significant difference compared with corresponding control density microdroplet value $(P<0.01)$

However, the proportion of zygotes that developed to the partially or fully hatched blastocyst stage in the ' $G \mathrm{O}^{\prime}$ system $(50.4 \%)$ was significantly higher than for culture in standard microdroplets $(7.6 \% ; P<0.0001)$, but was not different from that of the high density microdroplets (42\%). 'GO' blastocysts contained significantly more cells in total $(79.6 \pm 2.1)$ than did blastocysts grown in standard microdroplets $(68.7 \pm 2.0 ; P<0.01)$ but had a similar total number of cells to blastocysts grown in high density microdroplet cultures (85.8 \pm 2.7 ; Fig. 5). In a similar trend, ' $\mathrm{GO}$ ' blastocysts contained significantly more trophectoderm cells $(62.2 \pm 2.0)$ than did blastocysts derived from standard microdroplet culture $(52.7 \pm 1.7 ; P<0.01)$, but were similar in trophectoderm cell number to blastocysts derived from high density microdroplet cultures $(68.8 \pm 2.5)$. The number of ICM cells of 'GO' blastocysts was not different from that of blastocysts cultured in standard microdroplets or high density microdroplets $(17.4 \pm 0.5,16 \pm 0.5$ and $17 \pm 0.4$, respectively, Fig. 4).

\section{Discussion}

The results of the present study demonstrate that the 'GO' system provides a culture microenvironment suitable for development of mouse blastocysts and is not detrimental to the implantation potential of blastocysts relative to a traditional in vitro system.

In comparison to the standard microdroplet system of group preimplantation embryo culture (ten zygotes in $20 \mu \mathrm{l})$, the 'GO' system supported the development of similar proportions of blastocysts but larger proportions of partially or fully hatched blastocysts from the zygote stage. It is possible that the reduced volume of medium within the 'GO' system promotes the accumulation of secreted proteolytic enzymes that reportedly play a role in zona thinning of mouse (Perona and Wassarman, 1986; Sawada et al., 1990; Lee et al., 1997) and human embryos (Bongso and Fong, 1993). Such enzymes may also act in a paracrine manner, as shown by Mishra and Seshagiri (1998), whereby the zonae pellucidae of cleavage stage hamster embryos underwent degradation and thinning during group culture with hatching blastocysts. In the present study, a similar proportion of hatching blastocysts to that of ' $\mathrm{GO}$ '-derived blastocysts was observed after culture in microdroplets at the same zygote density as used in the 'GO' system. This result indicates that the action of proteolytic enzymes may be a function of concentration and can be achieved by increasing the embryo density or reducing the total volume of culture. However, the reduced volume of the 'GO' system demonstrates that the paracrine effect of increased concentrations of secretory enzymes can be elicited by as few as two preimplantation embryos.

'GO'-cultured blastocysts had significantly higher numbers of cells compared with standard microdroplet-derived control blastocysts in terms of total cell population, as well as subpopulations of ICM and trophectoderm cells. This increase was also observed in blastocysts that were grown from zygotes in microdroplets at the same zygote density as 'GO' cultures. It is possible that this mitogenic effect is a result of the concentration of putative embryotrophic factors at higher density embryo culture. Putative embryotrophic factors have been described previously (O'Niell, 1998). Preimplantation patterns of expression of peptide secretory factors and their receptors have been studied extensively (Kane et al., 1997), with a recent study providing evidence that active secretion of factors into the surrounding culture medium by mouse blastocysts occurs and can result in upregulation of development (Diaz-Cueto et al., 2000). Expression of such factors may also be upregulated when embryos are cultured in groups rather than singly (Larson and Kubisch, 1999) and this may result in improved embryo development. Conversely, there may be an environmental threshold beyond which mitogenic development of preimplantation embryos is inhibited, due to high concentrations of secretory products or metabolic byproducts or both (Lane and Gardner, 2000), particularly where there is minimal free diffusion of solutes and potential competition for nutrients.

The 'GO' system represents a change to the physical characteristics of the culture environment, notably in terms of reduced volume and increased embryo density. The beneficial effects of a relative reduction in medium volume and increase in embryo density on development and viability have been reported previously (Brinster, 1968; Lane and Gardner, 1992; Bavister, 1995). In a study of the effects of variable embryo density during culture, Lane and Gardner (1992) found that two-cell 
embryos cultured in groups to the morula stage contained significantly more cells than did morulae cultured individually from the two-cell stage in microdroplets. In addition, numbers of blastocyst cells were found to increase proportionally with increased embryo densities at three individual culture volumes $(5,20$ and $320 \mu \mathrm{l})$, but the number of cells reached a plateau at densities of $3.2,0.1$ and 0.025 embryos per $\mu \mathrm{l}$, respectively. Furthermore, Lane and Gardner (1992) investigated the effect of culture within variable volumes of media in 5-80 $\mu \mathrm{l}$ glass capillaries to eliminate the potential detrimental affects of oil in the culture system. Blastocysts that were cultured individually from the two-cell stage onwards in volumes of 5 and $10 \mu \mathrm{l}$ in capillaries had significantly higher numbers of cells than did those grown in equivalent microdroplet volumes. The results of the present study are difficult to compare with those of Lane and Gardner (1992) because of the use of a different mouse strain, the culture of zygotes and not two-cell embryos, and the use of fivefold lower volumes of medium. However, the results of the present study confirm the finding that total numbers of blastocyst cells are increased at higher embryo densities ( 0.5 embryos per $\mu$ l versus 2 embryos per $\mu \mathrm{l}$ ), although the embryos were not compared with those cultured individually. This increase was initially evident as a combination of increased numbers of ICM and trophectoderm cells. Results from the additional experiment including high density embryo culture in microdroplets revealed only a specific increase in numbers of trophectoderm cells, and it is possible that larger samples of blastocysts may be needed to determine whether numbers of ICM cells do indeed differ. During the initial determination of the optimal number of zygotes for culture in the 'GO' system to maximize embryo survival, a decrease in the proportion of blastocysts developing from zygotes (data not shown) was observed when zygotes were cultured in groups of three or more per capillary. This observation concurs with the plateau in development observed by Lane and Gardner (1992) at the density of 3.2 embryos per $\mu \mathrm{l}$ at the lowest volume of $5 \mu \mathrm{l}$. Uniquely, the present study demonstrates for the first time that as little as $1 \mu$ l of culture medium is sufficient to support blastocyst development from the zygote, a stage of development that is less amenable to culture than the two-cell stage (Lawitts and Biggers, 1991).

An additional factor that may be relevant to blastocyst quality after culture in the 'GO' system is the reduction in the amount of potentially embryotoxic materials to which the embryos are exposed. These elements include mineral oil (from $8 \mathrm{ml}$ in $60 \mathrm{~mm}$ Petri dishes to approximately $0.2 \mu \mathrm{l}$ as residual within a 'GO', which represents a $4 \times 10^{4}$-fold reduction) and medium (from $20 \mu \mathrm{l}$ to $<1 \mu \mathrm{l}$, which represents a 20 -fold reduction). Both oil and medium have been reported to carry chemical and microbial contaminants (Madison et al., 1991; Erbach et al., 1995; Cottell et al., 1996). In the 'GO' system, exposure to plastic cultureware, another element of culture systems that has been associated with embryotoxicity (Bavister, 1995), has been reduced, with most of the culture taking place within inert glass. Although all culture media, mineral oil and plastic cultureware used in these experiments had passed the mouse zygote bioassay (>70\% development to blastocyst), there still remains the possibility that trace amounts of embryotoxic material are permissive to development to the blastocyst stage, but have other undetectable effects on embryo development. Thus, a reduction in exposure to potentially embryotoxic elements in the culture system may be generally more permissive to survival. During optimization, it was found that mouse zygotes failed to develop to the blastocyst stage in $1 \mu \mathrm{l}$ microdroplets under mineral oil and development was arrested at early cleavage stages (data not shown). This finding supports the contention that the higher surface area to volume ratios of microdroplets of culture medium under oil may result in increased surface interactions (for example, loss of lipophilic factors into oil or transfer of toxic compounds from oil), as suggested by Lane and Gardner (1992). The putative toxic affects of mineral oil have been alleviated by washing and equilibration of oil in culture medium (Siddiquey and Cohen, 1982). However, it has been argued that the washing away of oil proven to be non-embryotoxic in the mouse zygote bioassay provides no additional improvement in outcome (Gardner and Lane, 2000). Despite the fact that the oil used in the present study was not cleaned in any way, there was no obvious detriment to the development of blastocysts in as little as $1 \mu$ l of medium in the 'GO' microenvironment, even when a small amount of residual oil was observed to be in direct physical contact with the blastocysts.

Subsequent to blastocyst development, no difference was observed between the rates of implantation or fetus formation after uterine transfer of blastocysts grown in the 'GO' system or in standard microdroplets. This finding may be explained by the potential similarities in number of ICM cells observed in the later culture experiment, despite the earlier observation of larger numbers of ICM cells in 'GO'-derived blastocysts. It is promising that, after implantation, morphologically normal blastocysts produced from 'GO' culture formed morphologically normal fetuses to a similar extent to standard microdroplet control blastocysts. However, both in vitro culture groups produced significantly lower numbers of viable fetuses than did in vivo derived blastocysts, a result which concurs with that of Lane and Gardner (1992).

In conclusion, culture of mouse zygotes in the 'GO' system supports blastocyst development in simple culture medium. The evidence of increased blastocyst cell division in the 'GO' microenvironment is probably due to the concentration of putative embryotrophic factors as a function of increased embryo density. Despite these developmental improvements, 'GO'-derived blastocysts 
remained comparable to standard microdroplet-derived blastocysts in terms of development after implantation. The 'GO' system represents an alternative to conventional culture systems in that it is potentially a more economical alternative to current embryo culture methods. Furthermore, the 'GO' culture system can effectively allow for embryo culture at high density even when very few embryos are available for culture, an application which may be of relevance to the culture of human embryos. The 'GO' system may also provide a basis for future research into the effects of engineering modifications of embryo containment and culture, such as microfluidic vessels.

The authors would like to thank Monash IVF for their financial support of this study and the animal storage facility staff at Monash University for their ongoing assistance.

\section{References}

Bavister BD (1995) Culture of preimplantation embryos: facts and artifacts Human Reproduction Update 191-148

Bongso A and Fong CY (1993) The effect of coculture on human zygote development Current Opinion in Obstetrics and Gynecology 5 585-593

Bongso A, Fong CY, Ng SC and Ratnam S (1993) The search for improved in vitro systems should not be ignored: embryo co-culture may be one of them Human Reproduction 8 1155-1160

Bongso TA, Fong CY, Ng CY and Ratnam SS (1994) Blastocyst transfer in human in vitro fertilization: the use of embryo co-culture Cell Biology International 18 1181-1189

Brinster RL (1968) Mammalian embryo culture. In The Mammalian Oviduct pp 419-444 Eds ESE Hafez and RJ Blandau. University of Chicago Press, Illinois

Cottell E, McMorrow J, Lennon B, Fawsy M, Cafferkey $M$ and Harrison RF (1996) Microbial contamination in an in vitro fertilization embryo transfer system Fertility and Sterility 66 776-780

Diaz-Cueto L, Stein P, Jacobs A, Schultz RM and Gerton GL (2000) Modulation of mouse preimplantation embryo development by acrogranin (epithelin/granulin precursor) Developmental Biology 217 406-418

Erbach GT, Bhatnagar P, Baltz JM and Biggers JD (1995) Zinc is a possible toxic contaminant of silicone oil in microdrop cultures of preimplantation mouse embryos Human Reproduction 103248 3254

Fong CY, Bongso A, Ng SC, Kumar J, Trounson A and Ratnam S (1998) Blastocyst transfer after enzymatic treatment of the zona pellucida: improving in vitro fertilization and understanding implantation Human Reproduction 13 2926-2932

Gardner DK (1998a) Changes in requirements and utilization of nutrients during mammalian preimplantation embryo development and their significance in embryo culture Theriogenology 49 83-102

Gardner DK (1998b) Development of serum-free media for the culture and transfer of human blastocysts Human Reproduction 13 Supplement $\mathbf{4}$ $218-225$

Gardner DK and Lane M (1993) Embryo culture systems. In Handbook of In Vitro Fertilization pp 85-114 Eds AO Trounson and DK Gardner. CRC Press, Boca Raton

Gardner DK and Lane M (1997) Culture and selection of viable blastocysts: a feasible proposition for human IVF? Human Reproduction Update 3 367-382

Gardner DK and Lane M (2000) Embryo culture systems. In Handbook of In Vitro Fertilization pp 205-264 Eds AO Trounson and DK Gardner. CRC Press, Boca Raton

Gardner DK and Leese HJ (1990) Concentrations of nutrients in mouse oviduct fluid and their effects on embryo development and metabolism in vitro. Journal of Reproduction and Fertility 88 361-368
Gardner DK, Lane M, Spitzer A and Batt PA (1994) Enhanced rates of cleavage and development for sheep zygotes cultured to the blastocyst stage in vitro in the absence of serum and somatic cells: amino acids, vitamins, and culturing embryos in groups stimulate development Biology of Reproduction $\mathbf{5 0} 390-400$

Hammitt DG, Walker DL, Syrop CH, Miller TM and Bennett MR (1991) Treatment of severe male-factor infertility with high concentrations of motile sperm by microinsemination in embryo cryopreservation straws Journal of In Vitro Fertilization and Embryo Transfer 8 101-110

Hogan B, Costantini F and Lacy E (1986) Manipulating the Mouse Embryo: A Laboratory Manual Cold Spring Harbor Laboratory, New York

Kane MT, Morgan PM and Coonan C (1997) Peptide growth factors and preimplantation development Human Reproduction Update 3 137-157

Lane M and Gardner DK (1992) Effect of incubation volume and embryo density on the development and viability of mouse embryos in vitro. Human Reproduction $7558-562$

Lane M and Gardner DK (2000) Lactate regulates pyruvate uptake and metabolism in the preimplantation mouse embryo Biology of Reproduction 62 16-22

Larson MA and Kubisch HM (1999) The effects of group size on development and interferon-tau secretion by in vitro fertilized and cultured bovine blastocysts Human Reproduction 14 2075-2079

Lawitts JA and Biggers JD (1991) Overcoming the 2-cell block by modifying standard components in a mouse embryo culture medium Biology of Reproduction 45 245-251

Lee DR, Lee JE, Yoon HS, Lee HJ, Kim MK and Roh SI (1997) The supplementation of culture medium with protease improves the hatching rate of mouse embryos Human Reproduction 12 2493-2498

Madison V, Greve T, Avery B and Wamberg T (1991) The effect of endotoxincontaminated medium on in vitro fertilization and development of bovine oocytes matured in vitro. Reproduction, Nutrition and Development 31 159-165

Magli MC, Gianaroli L, Ferraretti AP, Fortini D, Fiorentino A and D'Errico A (1995) Human embryo co-culture: results of a randomized prospective study International Journal of Fertility and Menopausal Studies $\mathbf{4 0} 254$ 259

Mishra A and Seshagiri PB (1998) Successful development in vitro of hamster 8-cell embryos to 'zona-escaped' and attached blastocysts: assessment of quality and trophoblast outgrowth Reproduction, Fertility and Development 10 413-420

Mulnard JG (1965) Studies of the regulation of mouse ova in vitro. In Preimplantation Stages of Pregnancy pp 123-138 Eds GEW Wolstenholme and $\mathrm{M} \mathrm{O}^{\prime}$ Connor. J \& A Churchill, London

$\mathbf{O}^{\prime}$ Niell C (1998) Autocrine mediators are required to act on the embryo by the 2-cell stage to promote normal development and survival of mouse preimplantation embryos in vitro. Biology of Reproduction 58 13031309

Paria BC and Dey SK (1990) Preimplantation embryo development in vitro: cooperative interactions among embryos and role of growth factors Proceedings National Academy of Sciences USA 87 4756-4760

Perona RM and Wassarman PM (1986) Mouse blastocysts hatch in vitro by using a trypsin-like proteinase associated with cells of mural trophectoderm Developmental Biology 114 42-52

Pincus G and Werthessen NT (1938) The comparative behaviour of mammalian eggs in vivo and in vitro. III: Factors controlling the growth of the rabbit blastocyst Journal of Experimental Zoology 78 1-38

Pruitt JA, Forrest DW, Burghardt RC, Evans JW and Kraemer DC (1991) Viability and ultrastructure of equine embryos following culture in a static or dynamic system Journal of Reproduction and Fertility Supplement $4 \mathbf{4}$ 405-410

Ranoux C and Seibel MM (1990) New techniques in fertilization: intravaginal culture and microvolume straw Journal of In Vitro Fertilization and Embryo Transfer 7 6-8

Sakkas D, Jaquenoud N, Leppens G and Campana A (1994) Comparison of results after in vitro fertilized human embryos are cultured in routine medium and in coculture on Vero cells: a randomized study Fertility and Sterility 61 521-525

Sawada H, Yamazaki K and Hoshi M (1990) Trypsin-like hatching protease from mouse embryos: evidence for the presence in culture 
medium and its enzymatic properties Journal of Experimental Zoology 254 83-87

Siddiquey AK and Cohen J (1982) In vitro fertilization in the mouse and the relevance of different sperm/egg concentrations and volumes Journal of Reproduction and Fertility 66 237-242

Thouas GA, Korfiatis NA, French AJ, Jones GM and Trounson AO (2002) Simplified technique for differential staining of inner cell mass and trophectoderm cells of mouse and bovine blastocysts Reproductive Biomedicine Online 3 25-29

Vajta G, Peura TT, Holm P, Paldi A, Greve T, Trounson AO and Callesen H (2000) New method for culture of zona-included or zona-free embryos: the Well of the Well (WOW) system Molecular Reproduction and Development 55 256-264

Van Blerkom J (1993) Development of human embryos to the hatched blastocyst stage in the presence or absence of a monolayer of Vero cells Human Reproduction 8 1525-1539 van der Ven HH, Hoebbel K, al-Hasani S, Diedrich K and Krebs D (1989) Fertilization of human oocytes in capillary tubes with very small numbers of spermatozoa Human Reproduction 4 72-76

Whitten WK and Biggers JD (1968) Complete development in vitro of the pre-implantation stages of the mouse in a simple chemically defined medium Journal of Reproduction and Fertility 17 399-401

Wilson JM, Zalesky DD, Looney CR, Bondioli KR and Magness RR (1992) Hormone secretion by preimplantation embryos in a dynamic in vitro culture system Biology of Reproduction 46 295-300

Received 21 November 2002.

First decision 20 December 2002.

Revised manuscript received 29 April 2003.

Accepted 1 May 2003. 